\title{
Log-normal flux distribution of bright Fermi blazars
}

\author{
Z. Shah ${ }^{1}$, N. Mankuzhiyil ${ }^{2}$, A. Sinha ${ }^{3,4}$, R. Misra $^{3}$, S. Sahayanathan ${ }^{2}$ and N. Iqbal ${ }^{1}$ \\ 1 Department Of Physics, University of Kashmir, Srinagar-190006, India; shahzahir4@ gmail.com \\ 2 Astrophysical Sciences Division, Bhabha Atomic Research Centre, Mumbai-400085, India; \\ nijil@barc.gov.in \\ ${ }^{3}$ Inter-University Center for Astronomy and Astrophysics, Pune-411007, India \\ 4 now at: AstroParticule et Cosmoligie, CNRS/University Paris Diderot, 10 Rue Alice Domon et Leonie \\ Duquet, Paris 75013
}

\begin{abstract}
We present the results of the $\gamma$-ray flux distribution study on the brightest blazars which are observed by the Fermi-LAT. We selected 50 brightest blazars based on the maximum number of detection reported in the LAT third AGN catalog. We performed standard unbinned maximum likelihood analysis on the LAT data during the period between August 2008 and December 2016, in order to obtain the average monthly flux. After quality cuts, blazars for which at least $90 \%$ of the total flux was survived were selected for the further study, and this includes 19 FSRQs and 19 BL Lacs. The Anderson-Darling and $\chi^{2}$ tests suggest that the integrated monthly flux follow a log-normal distribution for all sources, except for three FSRQs for which neither a normal nor a log-normal distribution was preferred. A double log-normal flux distribution tendency were observed in these sources, though it has to be confirmed with improved statistics. We also found that, the standard deviation of the lognormal flux distribution increases with the mean spectral index of the blazar, and can be fitted with a line of slope $0.24 \pm 0.04$. We repeat our study on three additional brightest unclassified blazars to identify their flux distribution properties. Based on the features of their log-normal flux distribution, we infer these unclassified blazars may be closely associated with FSRQs. We also highlight that considering the log-normal behavior of the flux distribution of blazars, averaging their long term flux in linear scale can largely under estimate the nominal flux and this discrepancy can propagate down to the estimation of source parameters through spectral modeling.
\end{abstract}

Key words: Active Galaxy: Blazar, FSRQ, BL Lac, gamma-rays

\section{INTRODUCTION}

Blazars are subclass of Active Galactic Nuclei (AGNs) with their relativistic jets pointing towards line of sight of the observer (Blandford \& Königl 1979). Even though the mechanism behind the formation 
of relativistic jets is not fully understood yet, it is most likely related to the focusing properties of the fully ionized, rotating accretion disk (Blandford \& Znajek 1977). Blazars include BL Lac objects and FlatSpectrum Radio Quasars (FSRQs), where the significant difference between the two classes being their optical emission/absorption lines, which are strong for FSRQs, while weak or absent for BL Lacs (Urry \& Padovani 1995).

The spectral energy distribution (SED) of blazars consists of two broad emission components, where the low energy component peaks at optical to X-ray band, while the high energy component peaks at $\mathrm{MeV}$ to $\mathrm{TeV}$ band. BL Lac objects are further subdivided based on the peak frequency $\left(\nu_{s}\right)$ of their low energy component namely, high energy peaked BLLac $\left(\mathrm{HBL} ; \nu_{s}>10^{15.3} \mathrm{~Hz}\right)$, intermediate energy peaked BL Lac (IBL; $10^{14}<\nu_{s} \leq 10^{15.3} \mathrm{~Hz}$ ), and low energy peaked BLLac (LBL; $\nu_{\mathrm{s}} \leq 10^{14} \mathrm{~Hz}$ ) (Fan et al. 2016). In case of FSRQs, $\nu_{s}$ usually falls at relatively lower frequencies $\left(\lesssim 10^{14} \mathrm{~Hz}\right)$. The low energy component of the blazar SED is commonly attributed to the synchrotron emission due to the interaction of relativistic electrons in the jet magnetic field; whereas the high energy component is explained as inverse Compton (IC) scattering process. If the target low energy photons for the IC process is the synchrotron photon itself then the IC mechanism is called Synchrotron Self Compton (SSC; Marscher \& Gear 1985; Band \& Grindlay 1985). On the other hand, if the photon origin is external to the jet, e.g. broad line region (BLR), obscuring torus, Cosmic Microwave Background (CMB) etc., then the process is called external Compton (EC) mechanism (Dermer et al. 1992; Sikora et al. 1994; Shah et al. 2017)). Alternate to this leptonic interpretation of the high energy emission, hadronic models involving nuclear cascades were also put forth and are successful in explaining many observed features of blazars (Mannheim \& Biermann 1992; Böttcher 2007).

One of the distinct property of blazars is their rapid flux and spectral variability across the entire electromagnetic spectrum on time scales ranging from minutes to years. Though the cause of variability is still not well understood, plausible clues can be obtained by studying the long term flux distribution of blazars. Such studies have been performed in detail at X-ray energies for Seyfert galaxies and X-ray binaries, where the emission at these energies is dominated by the accretion disk or its corona. The X-ray flux of Seyfert 1 IRAS 13224-3809 using ASCA observations in different epochs, exhibit a log-normal distribution (Gaskell 2004). In another study, Uttley et al. (2005) found that the X-ray flux of Seyfert 1 NGC 4051 also shows a log-normal distribution, which was comparable to the X-ray flux of the black-hole X-ray binary Cyg X1. Linear relationship between the optical flux and the corresponding variation were noticed in Seyfert 1 NGC 4151 (Lyutyj \& Oknyanskij 1987), which in turn is an indication of log-normality of flux distribution. A similar relationship was also noticed in X-ray band in both Seyfert 1 Mrk 766 (Vaughan et al. 2003b), and Seyfert 2 MCG 6-30-15 (Vaughan et al. 2003a). The log-normality of flux distribution in a blazar was first detected in BL Lacertae, from the RXTE observations (Giebels \& Degrange 2009). This result is particularly interesting since for blazars, X-ray emission originate from jets rather than the accretion disk or its environment. Hence, this result may hint the plausible disk-jet connection in blazars, which is still not clearly understood. The log-normality was later observed in many blazars at different energies. For instance, such behavior was inferred in Mrk 421 and Mrk 501 at Very High Energy (VHE >100 GeV) band, though the data was noncontinuous (Tluczykont et al. 2010). Similarly, the 4-year flux distribution of blazars given 
in the third Fermi-LAT catalog of AGNs (3LAC; Ackermann et al. (2015)), showed a log-normal behaviour. While quantifying the flux variability in Mrk 421, Sinha et al. (2016) also noticed a log-normal flux distribution (more than normal) trend, through out the frequencies from radio to VHE. On the contrary, a detailed multi-wavelength study of FSRQ PKS 1510-089, Kushwaha et al. (2016) found that the flux distribution follow two distinctive log-normal profiles in both optical and $\gamma$-rays, while X-ray flux distribution follow a single log-normal distribution. Interestingly, the $\gamma$-ray flux distribution of the same source, obtained from a near continuous data during August 2008-October 2015, was well fitted by a log-normal distribution and similar was the case of HBL Mrk 421 and FSRQs B2 1520+31. On the other hand, the $\gamma$-ray flux distribution of FR I radio galaxy NGC 1275 was not able to be represented by a log-normal or normal function, even though the rms increases linearly with flux (Kushwaha et al. 2017).

In this work, we aim to study the flux distribution properties of the brightest Fermi blazars using the data collected in more than 8 years. We also investigate the associated spectral properties of these brightest blazars. Further, we examine the above properties in order to associate the unclassified blazar types (BCUs) with the known blazar classes. We select bright blazars from the 3LAC, and analyze the data (described in Sect. 2). In order to overcome the effect of short-term flux variations, which are most likely associated with the change in the emission region geometry, we consider the flux in monthly bins for our study. After analyzing the features of the flux distribution, and verifying the log-normality (Sect. 3), we study the association of flux distribution with spectral properties (Sect.4). The results and possible implications are discussed in Sect. 5 .

\section{FERMI-LAT ANALYSIS}

The Large Area Telescope (LAT) on board Fermi satellite is a pair conversion detector (Atwood et al. 2009 ) with an effective area $\sim 8000 \mathrm{~cm}^{2} / \mathrm{GeV}$ photon, and field of view $\sim 2.4 \mathrm{sr}$, in the energy range from $20 \mathrm{MeV}$ to more than $300 \mathrm{GeV}$, which scans entire sky in every 3 hours. We made a primary selection of 25 FSRQs and 25 BL Lacs from the four year NASA'S Fermi 3LAC interactive table ${ }^{1}$. The selection was based on the criteria such that the chosen FSRQs and BL Lacs should have monthly averaged photon flux $>6.5 \times 10^{-9}$ photons $\mathrm{cm}^{-2} \mathrm{~s}^{-1}$ and $>5.5 \times 10^{-9}$ photons $\mathrm{cm}^{-2} \mathrm{~s}^{-1}$ respectively, and the number of upper limits (i.e, non detections) should be less than or equal to 4 . We have then downloaded the first 8.4 years of data ( from 2008 August to 2016 December) for the selected sources. The data were analyzed in the energy range from $100 \mathrm{MeV}$ to $500 \mathrm{GeV}$, in a region of interest (ROI) of $10^{\circ}$ centering the nominal source positions. The analysis was carried out using the maximum likelihood method (gtlike) and standard Fermi SCIENCE TOOLS (version v9r12) with the instrument response function 'P8R2_SOURCE_V6', Galactic diffuse model 'gll_iem_v06.fit' and isotropic background model 'iso_p8R2_SOURCE_V6_v06.txt'. Events which were contaminated by the bright Earths limb were excluded using zenith angle cut of $90^{\circ}$. Further the time bins with $T S<9$ were excluded, which correspond to a detection significance of $\sqrt{T S} \approx 3 \sigma$ We estimated monthly photon flux, energy flux, and spectral index for all the sources using the maximum likelihood analysis.

1 https://fermi.gsfc.nasa.gov/ssc/data/access/lat/4yr_- catalog/3FGL-table/ 


\section{FLUX DISTRIBUTION}

The monthly average $\gamma$-ray flux obtained in the analysis of $\sim 100$ months of data were distributed to a histogram of fluxes, for each source. An adaptive binning was used for each source to ensure the bin width is larger than the average error of the flux within a bin. Apart from the flux that corresponds to a TS value $T S \leq 9$, we have also excluded the flux with larger uncertainty, such that $\mathrm{F} / \delta \mathrm{F}<2$. In order to avoid the bias due to a possible lack of lower luminosity flux states, we restrict our focus only on the blazars, for which the total excluded flux points (after the cuts mentioned above) are less than $10 \%$. After this cut, 38 (out of 50) blazars survived, which include 19 BL Lacs and 19 FSRQs.

We fit all 38 flux histograms in log-scale, with functions

$$
\mathrm{L}(\mathrm{x})=\frac{1}{\sqrt{2 \pi} \sigma} \exp \frac{\frac{-(\mathrm{x}-\mu)^{2}}{2 \sigma^{2}}}{[\log -\text { normal distribution }]}
$$

and

$$
\mathrm{G}(\mathrm{X})=\frac{1}{\sqrt{2 \pi} \sigma} \exp ^{\frac{\left(10^{\mathrm{x}}-\mu\right)^{2}}{2 \sigma^{2}}} 10^{\mathrm{x}} \log _{\mathrm{e}}(10) \quad \text { [normal distribution] }
$$

where $\sigma$ and $\mu$ are the standard deviation and mean of the distribution, respectively.

The flux histograms of the blazars are plotted in log-scale in Fig.1. The normal and log-normal fits are shown as red and blue lines respectively. Fig. 2 (left panel) shows the comparison between the reduced $\chi^{2}$ obtained from normal and log-normal distributions. The fit parameters together with the computed skewness for both distributions are shown in Table 1 . The flux distributions are found to be significantly skewed, whereas the skewness of the log of flux distribution is consistent with zero, thus suggesting log-normal trend (Fig. 2, right panel). Apart from calculating the reduced $\chi^{2}$, we have also performed Anderson-Darling (AD) test, in order to verify the normality/log-normality of the fits. The reduced $\chi^{2}$ of the fit, together with the AD test statistics and rejection/null hypothesis probability (p-value) are also shown in the Table 1 .

We note that the reduced $\chi^{2}$ for the normal flux distribution of some of the blazars fall in a reasonable range. However, the $\mathrm{p}$-value estimated from the AD test rejects the normal distribution $(\mathrm{p}<0.05)$ for all sources, except for the FSRQ J0957.6+5523 (with a high reduced $\chi^{2}$ in this case). On the other hand, the $\mathrm{AD}$ test p-value, and the reduced $\chi^{2}$ of the flux distribution of J0957.6+5523 do not reject the lognormal distribution of flux either. It is interesting to note that the $\chi^{2}$ and AD tests do not reject the lognormality of the flux distribution of most of the blazars. Nevertheless, both tests reject the log-normality of the flux distribution of FSRQs J2329.3-4955, J1504.4+1029 and J1625.7-2527. Even though the AD tests marginally (eg: J1427.9-4206), and completely (J1512.8-0906) reject the log-normal fits of a few blazar flux distributions, the corresponding $\chi^{2}$ values are reasonable enough not to reject the $\log$-normality.

The standard deviation obtained from the log-normal fits, which is a measure of flux variability, is comparatively high for FSRQs. In the case of BL Lacs, HBLs show a lower variability, while the variability of LBLs are similar to that of FSRQs. The variability of IBLs roughly fall in between HBLs and LBLs. The mean values of the standard deviation obtained from the log-normal fit of the considered FSRQs, HBLs, IBLs and LBLs are $0.41 \pm 0.11,0.21 \pm 0.04,0.27 \pm 0.03$, and $0.37 \pm 0.10$ respectively. We have also noticed that the standard deviation of the flux distribution of J0957.6+5523 is significantly smaller compared to other FSRQs, and could be treated as a steady FSRQ. 
Table 1 The fitting parameters of log-normal (column: 2, 3) and normal (column: 7, 8) flux distribution of Blazars. The computed skewness (column 4 and 9), reduced $\chi^{2}$ (column 5 and 10), and AD statistics (column 6 and 11), for both distributions are also shown.

\begin{tabular}{|c|c|c|c|c|c|c|c|c|c|c|}
\hline \multirow{3}{*}{$\begin{array}{l}\text { Blazar } \\
\text { Name }\end{array}$} & \multicolumn{10}{|c|}{ FSRQ } \\
\hline & \multicolumn{5}{|c|}{ Log-normal } & \multicolumn{5}{|c|}{ Normal } \\
\hline & width & centroid & skewness $(\kappa)$ & $\chi^{2} / d o f$ & $\mathrm{AD}$ (prob) & width $^{*}$ & centroid ${ }^{*}$ & skewness* & $\chi^{2} / d o f$ & $\mathrm{AD}$ (prob) \\
\hline J0457.0-2324 & $0.26 \pm 0.03$ & $-3.96 \pm 0.04$ & $-0.52 \pm 0.40$ & 2.21 & $0.64(0.09)$ & $8.33 \pm 1.40$ & $11.1 \pm 1.60$ & $0.71 \pm 0.40$ & 1.12 & $1.50(6.7 \mathrm{e}-04)$ \\
\hline J0730.2-1141 & $0.30 \pm 0.02$ & $-4.17 \pm 0.02$ & $-0.08 \pm 0.39$ & 0.42 & $0.37(0.41)$ & $6.39 \pm 1.17$ & $6.71 \pm 2.01$ & $1.40 \pm 0.39$ & 2.88 & $3.32(2.3 e-08)$ \\
\hline J0957.6+5523 & $0.12 \pm 0.01$ & $-4.24 \pm 0.01$ & $-0.14 \pm 0.39$ & 0.53 & $0.22(0.84)$ & $1.54 \pm 0.21$ & $5.85 \pm 0.28$ & $0.62 \pm 0.39$ & 2.89 & $0.69(0.07122)$ \\
\hline J1127.0-1857 & $0.45 \pm 0.04$ & $-4.49 \pm 0.06$ & $0.33 \pm 0.39$ & 0.67 & $0.72(0.06)$ & $6.48 \pm 2.04$ & $3.17 \pm 3.18$ & $1.83 \pm 0.39$ & 7.38 & $6.40(7.7 \mathrm{e}-16)$ \\
\hline $\mathrm{J} 1224.9+2122$ & $0.57 \pm 0.02$ & $-4.17 \pm 0.03$ & $0.17 \pm 0.39$ & 0.16 & $0.33(0.51)$ & $23.8 \pm 8.34$ & $8.26 \pm 17.6$ & $3.41 \pm 0.39$ & 11.7 & $10.97(<2.2 \mathrm{e}-16)$ \\
\hline J1246.7-2547 & $0.30 \pm 0.03$ & $-4.45 \pm 0.04$ & $0.31 \pm 0.39$ & 0.93 & $0.72(0.06)$ & $3.79 \pm 1.08$ & $3.25 \pm 1.50$ & $1.34 \pm 0.39$ & 5.21 & $5.44(1.6 \mathrm{e}-13)$ \\
\hline J1427.9-4206 & $0.33 \pm 0.02$ & $-3.75 \pm 0.03$ & $-0.88 \pm 0.40$ & 0.75 & $1.02(0.01)$ & $15.2 \pm 3.28$ & $19.6 \pm 5.39$ & $1.78 \pm 0.40$ & 1.73 & $4.61(1.6 \mathrm{e}-11)$ \\
\hline J1512.8-0906 & $0.37 \pm 0.04$ & $-3.73 \pm 0.05$ & $-0.80 \pm 0.38$ & 0.71 & $2.44(3.2 \mathrm{e}-06)$ & $15.7 \pm 4.52$ & $17.6 \pm 6.85$ & $2.27 \pm 0.38$ & 5.26 & $10.36(<2.2 \mathrm{e}-16)$ \\
\hline J0237.9+2848 & $0.33 \pm 0.02$ & $-4.43 \pm 0.02$ & $0.59 \pm 0.39$ & 0.36 & $0.71(0.06)$ & $3.04 \pm 0.77$ & $3.78 \pm 1.22$ & $3.39 \pm 0.39$ & 3.11 & $5.44(1.6 \mathrm{e}-13)$ \\
\hline J2254.0+1608 & $0.60 \pm 0.10$ & $-3.37 \pm 0.12$ & $-0.57 \pm 0.60$ & 1.44 & $0.83(0.03)$ & $78.7 \pm 47.1$ & $66.4 \pm 96.9$ & $2.42 \pm 0.60$ & 2.92 & $4.65(9.7 e-12)$ \\
\hline $\mathrm{J} 1522.1+3144$ & $0.27 \pm 0.02$ & $-4.07 \pm 0.02$ & $-1.02 \pm 0.39$ & 0.84 & $0.62(0.10)$ & $5.58 \pm 0.65$ & $9.13 \pm 0.96$ & $1.29 \pm 0.39$ & 0.90 & $2.11(2.1 \mathrm{e}-05)$ \\
\hline J1635.2+3809 & $0.38 \pm 0.03$ & $-4.14 \pm 0.03$ & $0.27 \pm 0.39$ & 0.72 & $0.76(0.05)$ & $11.1 \pm 3.45$ & $6.49 \pm 5.65$ & $1.71 \pm 0.39$ & 7.54 & $6.78(<2.2 \mathrm{e}-16)$ \\
\hline J2329.3-4955 & $0.54 \pm 0.10$ & $-3.97 \pm 0.14$ & $-0.24 \pm 0.39$ & 3.88 & $1.09(0.01)$ & $21.7 \pm 6.67$ & $15.6 \pm 12.9$ & $1.90 \pm 0.39$ & 6.33 & $4.16(2.0 \mathrm{e}-10)$ \\
\hline $\mathrm{J} 2345.2-1554$ & $0.51 \pm 0.03$ & $-4.10 \pm 0.04$ & $-0.29 \pm 0.40$ & 0.56 & $0.22(0.84)$ & $14.5 \pm 5.63$ & $10.7 \pm 9.85$ & $2.09 \pm 0.40$ & 5.70 & $6.66(<2.2 \mathrm{e}-16)$ \\
\hline J0808.2-0751 & $0.45 \pm 0.05$ & $-4.53 \pm 0.06$ & $0.51 \pm 0.41$ & 0.77 & $0.65(0.09)$ & $2.72 \pm 0.78$ & $2.71 \pm 1.25$ & $2.75 \pm 0.41$ & 4.42 & $8.46(<2.2 \mathrm{e}-16)$ \\
\hline J1229.1+0202 & $0.34 \pm 0.04$ & $-4.28 \pm 0.04$ & $0.57 \pm 0.38$ & 1.48 & $0.70(0.06)$ & $3.89 \pm 1.25$ & $5.29 \pm 1.93$ & $3.50 \pm 0.38$ & 3.12 & $12.89(<2.2 \mathrm{e}-16)$ \\
\hline J1256.1-0547 & $0.34 \pm 0.02$ & $-3.94 \pm 0.02$ & $0.29 \pm 0.37$ & 0.38 & $0.27(0.68)$ & $9.92 \pm 2.83$ & $11.9 \pm 4.16$ & $3.86 \pm 0.37$ & 3.01 & $8.63(<2.2 \mathrm{e}-16)$ \\
\hline J1504.4+1029 & $0.63 \pm 0.10$ & $-4.38 \pm 0.15$ & $0.14 \pm 0.38$ & 2.22 & $0.98(0.01)$ & $17.9 \pm 7.48$ & $5.29 \pm 11.4$ & $1.84 \pm 0.38$ & 11.7 & $8.54(<2.2 \mathrm{e}-16)$ \\
\hline \multirow[t]{2}{*}{ J1625.7-2527 } & $0.30 \pm 0.04$ & $-4.28 \pm 0.05$ & $0.47 \pm 0.45$ & 2.12 & $1.11(0.01)$ & $5.96 \pm 1.88$ & $4.64 \pm 2.98$ & $1.94 \pm 0.45$ & 5.71 & $6.87(<2.2 \mathrm{e}-16)$ \\
\hline & \multicolumn{10}{|c|}{ BL Lac } \\
\hline J0222.6+4301 & $0.23 \pm 0.02$ & $-4.04 \pm 0.02$ & $0.45 \pm 0.38$ & 0.50 & $0.44(0.28)$ & $5.23 \pm 1.07$ & $8.88 \pm 1.42$ & $2.35 \pm 0.38$ & 2.30 & $3.55(6.1 \mathrm{e}-09)$ \\
\hline J0238.6+1636 & $0.56 \pm 0.06$ & $-4.42 \pm 0.08$ & $0.30 \pm 0.42$ & 0.67 & $0.85(0.03)$ & $5.19 \pm 2.04$ & $3.57 \pm 2.97$ & $2.98 \pm 0.42$ & 6.97 & $7.70(<2.2 \mathrm{e}-16)$ \\
\hline J0428.6-3756 & $0.33 \pm 0.03$ & $-3.95 \pm 0.04$ & $-0.90 \pm 0.38$ & 1.84 & $11.45(0.01)$ & $9.75 \pm 2.14$ & $11.5 \pm 2.83$ & $1.03 \pm 0.38$ & 1.47 & $2.15(1.7 \mathrm{e}-05)$ \\
\hline J0538.8-4405 & $0.45 \pm 0.06$ & $-3.48 \pm 0.08$ & $0.42 \pm 0.38$ & 1.30 & $1.02(0.01)$ & $73.1 \pm 27.3$ & $30.5 \pm 40.2$ & $2.25 \pm 0.38$ & 10.5 & $8.04(<2.2 \mathrm{e}-16)$ \\
\hline J0721.9+7120 & $0.31 \pm 0.02$ & $-3.96 \pm 0.03$ & $-0.37 \pm 0.38$ & 1.05 & $0.66(0.08)$ & $9.66 \pm 1.75$ & $11.1 \pm 2.56$ & $1.03 \pm 0.38$ & 1.94 & $2.25(9.7 \mathrm{e}-06)$ \\
\hline J1104.4+3812 & $0.21 \pm 0.02$ & $-3.39 \pm 0.02$ & $-0.42 \pm 0.38$ & 0.91 & $0.48(0.22)$ & $23.0 \pm 1.89$ & $45.1 \pm 3.15$ & $1.67 \pm 0.38$ & 0.92 & $2.67(8.9 \mathrm{e}-07)$ \\
\hline $\mathrm{J} 1427.0+2347$ & $0.14 \pm 0.01$ & $-4.00 \pm 0.01$ & $-0.02 \pm 0.38$ & 0.20 & $0.34(0.50)$ & $3.74 \pm 0.56$ & $9.92 \pm 0.68$ & $0.61 \pm 0.38$ & 1.78 & $1.13(0.01)$ \\
\hline J1555.7+1111 & $0.17 \pm 0.01$ & $-3.84 \pm 0.01$ & $-0.04 \pm 0.38$ & 0.22 & $0.5124(0.19)$ & $6.88 \pm 0.78$ & $14.6 \pm 0.97$ & $1.00 \pm 0.38$ & 1.04 & $2.12(2.1 \mathrm{e}-05)$ \\
\hline J2158.8-3013 & $0.18 \pm 0.01$ & $-3.38 \pm 0.02$ & $0.25 \pm 0.38$ & 0.75 & $0.1918(0.89)$ & $19.1 \pm 2.52$ & $42.2 \pm 2.75$ & $2.17 \pm 0.38$ & 0.86 & $2.68(8.4 \mathrm{e}-07)$ \\
\hline J0112.1+2245 & $0.31 \pm 0.01$ & $-4.42 \pm 0.01$ & $-0.05 \pm 0.38$ & 0.13 & $0.14(0.97)$ & $4.06 \pm 0.76$ & $3.50 \pm 1.31$ & $1.70 \pm 0.38$ & 3.19 & $4.21(9.2 \mathrm{e}-11)$ \\
\hline J0449.4-4350 & $0.23 \pm 0.02$ & $-4.02 \pm 0.03$ & $-0.44 \pm 0.38$ & 1.57 & $0.51(0.20)$ & $6.35 \pm 0.80$ & $9.55 \pm 0.93$ & $0.75 \pm 0.38$ & 0.89 & $1.11(0.01)$ \\
\hline J0509.4+0541 & $0.25 \pm 0.02$ & $-4.36 \pm 0.02$ & $0.14 \pm 0.39$ & 0.69 & $0.19(0.89)$ & $3.63 \pm 0.95$ & $3.65 \pm 1.27$ & $1.53 \pm 0.39$ & 4.44 & $3.02(1.2 \mathrm{e}-07)$ \\
\hline J0818.2+4223 & $0.20 \pm 0.01$ & $-4.52 \pm 0.02$ & $-0.12 \pm 0.38$ & 0.95 & $0.26(0.72)$ & $1.61 \pm 0.16$ & $3.02 \pm 0.18$ & $0.91 \pm 0.38$ & 0.72 & $2.05(3.0 \mathrm{e}-05)$ \\
\hline $\mathrm{J} 1015.0+4925$ & $0.22 \pm 0.01$ & $-4.09 \pm 0.01$ & $0.26 \pm 0.38$ & 0.34 & $0.31(0.56)$ & $4.45 \pm 0.66$ & $8.11 \pm 0.97$ & $2.80 \pm 0.38$ & 1.36 & $3.94(7.1 \mathrm{e}-10)$ \\
\hline $\mathrm{J} 1058.5+0133$ & $0.25 \pm 0.03$ & $-4.43 \pm 0.04$ & $-0.31 \pm 0.39$ & 1.24 & $0.45(0.27)$ & $2.70 \pm 0.43$ & $3.83 \pm 0.41$ & $0.82 \pm 0.39$ & 1.09 & $2.67(8.6 \mathrm{e}-07)$ \\
\hline J1653.9+3945 & $0.28 \pm 0.02$ & $-2.88 \pm 0.03$ & $-0.48 \pm 0.38$ & 0.98 & $0.63(0.10)$ & $9.33 \pm 1.22$ & $13.6 \pm 1.58$ & $0.86 \pm 0.38$ & 1.06 & $1.30(2.1 \mathrm{e}-3)$ \\
\hline $\mathrm{J} 2202.7+4217$ & $0.35 \pm 0.03$ & $-4.02 \pm 0.04$ & $-0.14 \pm 0.38$ & 0.81 & $0.74(0.05)$ & $11.6 \pm 3.02$ & $9.69 \pm 4.56$ & $1.09 \pm 0.38$ & 4.85 & $2.82(3.8 \mathrm{e}-07)$ \\
\hline $\mathrm{J} 2236.5-1432$ & $0.41 \pm 0.03$ & $-4.51 \pm 0.04$ & $0.54 \pm 0.42$ & 0.68 & $0.63(0.10)$ & $7.91 \pm 2.48$ & $2.23 \pm 4.92$ & $3.63 \pm 0.42$ & 8.65 & $12.24(<2.2 \mathrm{e}-16)$ \\
\hline J0521.7+2113 & $0.28 \pm 0.02$ & $-4.05 \pm 0.02$ & $0.45 \pm 0.46$ & 0.34 & $0.38(0.39)$ & $6.06 \pm 1.26$ & $8.30 \pm 1.66$ & $2.41 \pm 0.46$ & 1.54 & $3.69(2.6 \mathrm{e}-09)$ \\
\hline
\end{tabular}

* units of $10^{-5} \mathrm{MeV} \mathrm{cm}^{-2} \mathrm{~s}^{-1}$ 

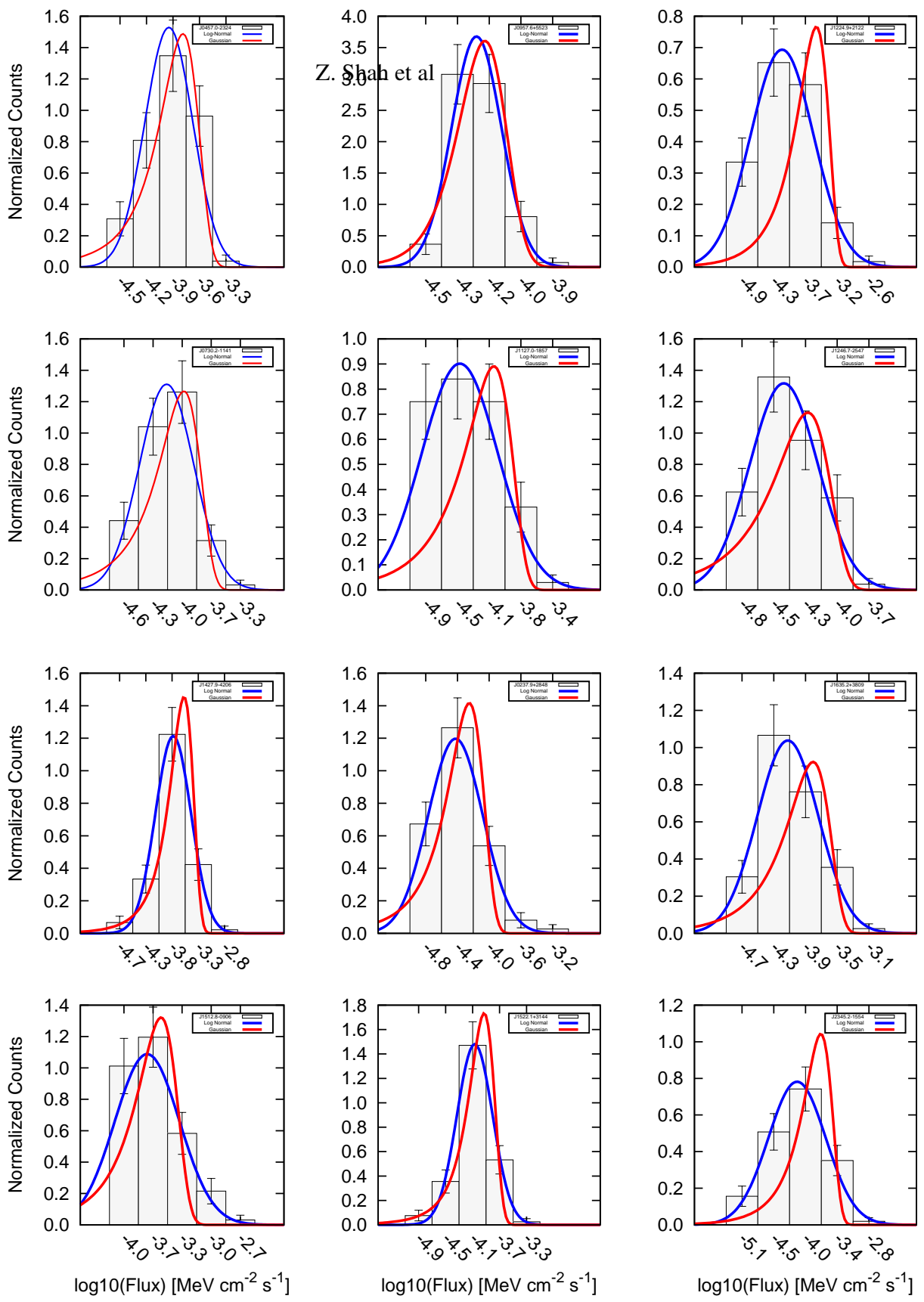

Fig. 1 Flux distribution of bright blazars in $\gamma$-ray band. The blue and red lines correspond to log-normal and normal fit respectively.

\subsection{The case of Uncertain type blazars}

More than 500 sources were classified as blazars of uncertain type (BCU) in 3LAC. Even though these sources are associated with extra-galactic counterparts, and show some of the blazar characteristics, they lack reliable classification based on spectral information. In order to investigate the flux distribution properties of such sources, we analyzed the long term data (in the same time period as of the other bright blazars) of three brightest BCUs (namely, J0522.9-3628, J0532.0-4827, and J1328.9-5608). These sources are comparatively less brighter than the known classified blazars that we considered. After analyzing the Fermi-LAT 

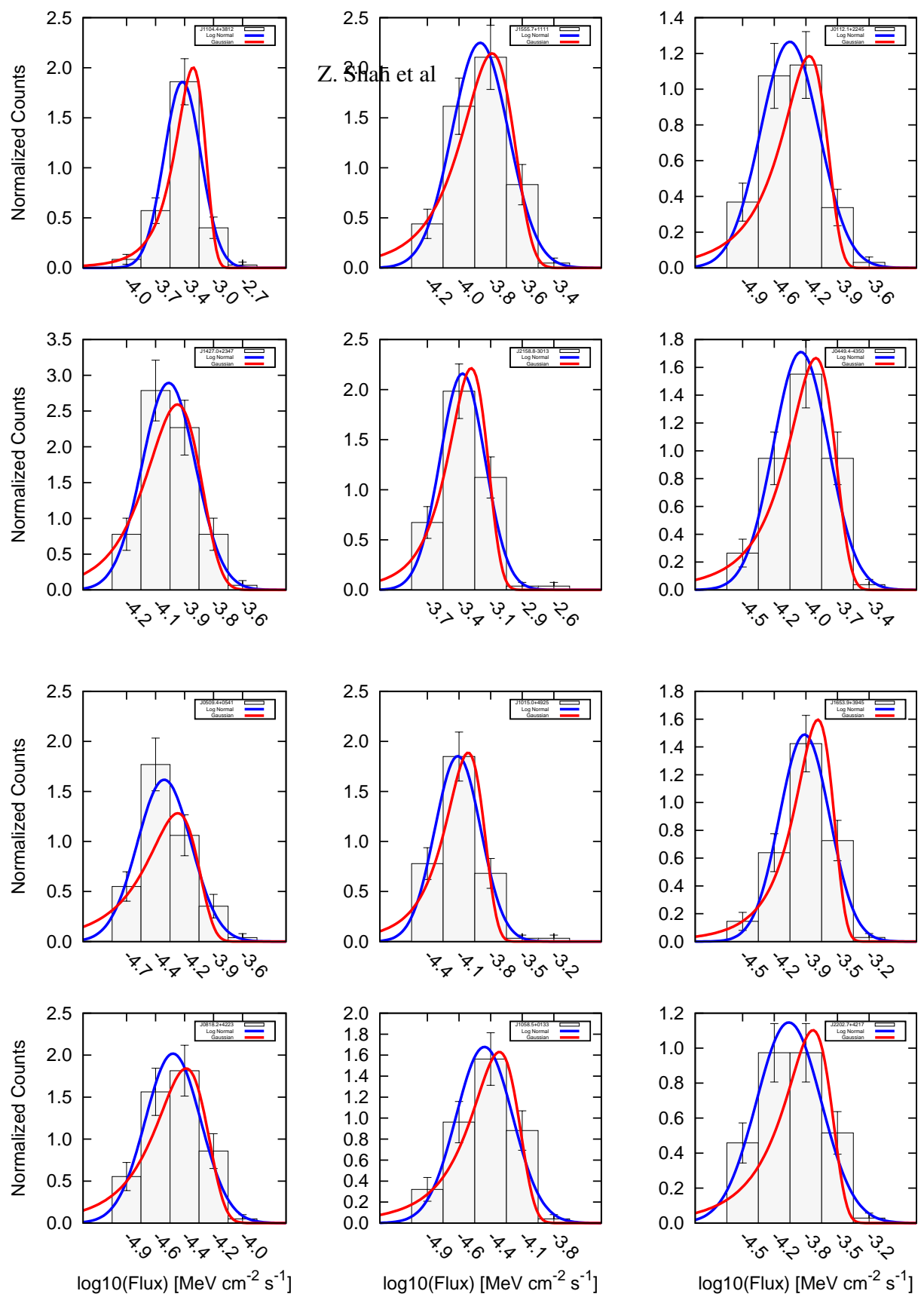

Fig. 1 (Continued)

for the sources (in the same order as above) are $0.78,0.80$ and 0.17 (instead of $6.72 \times 10^{-15}, 2.86 \times 10^{-16}$ and $2.37 \times 10^{-11}$ for normal distribution), which also propose log-normal distributions of flux over normal distributions. The obtained standard deviation from the log-normal fit of J0522.9-3628 and J1328.9-5608 are 0.29 and 0.34 respectively, while it is high $(0.47$, which is similar to FSRQs and LBLs) for the source J0532.0-4827. 

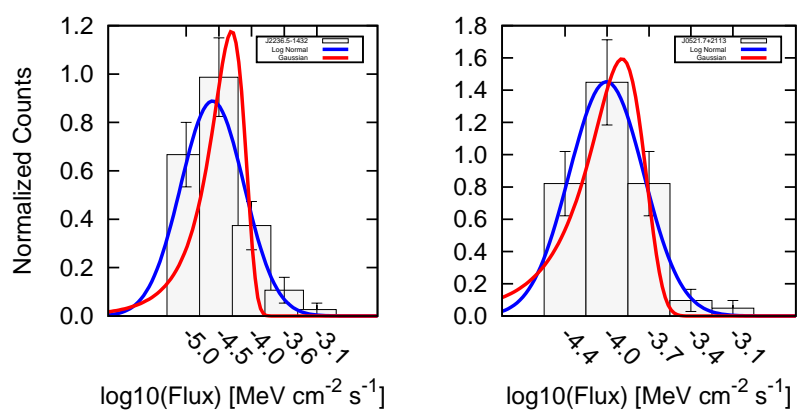

Fig. 1 (Continued)
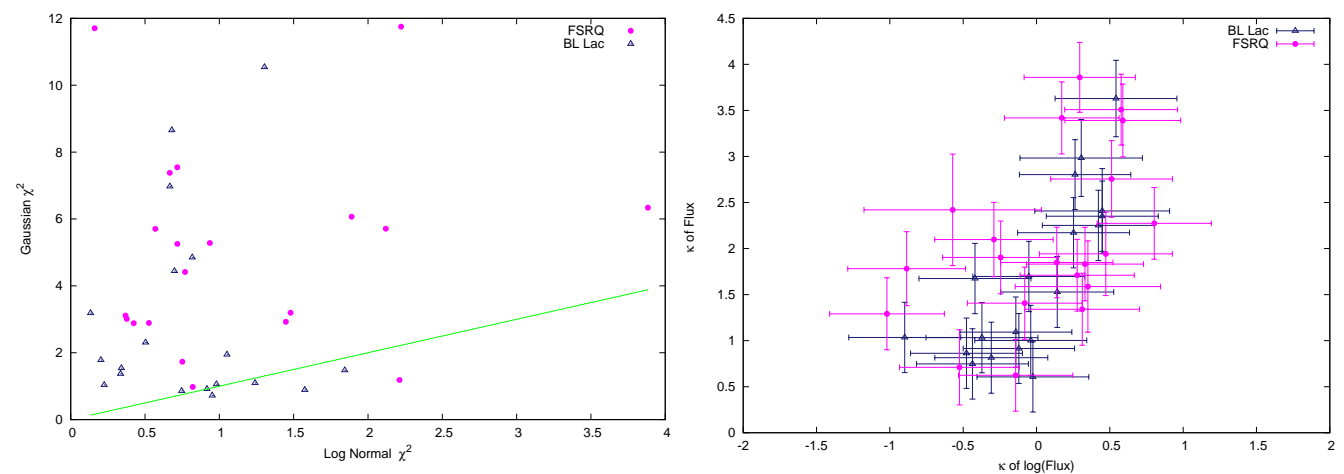

Fig. 2 Left: the reduced $\chi^{2}$ obtained from the blazar flux distribution by fitting log-normal distribution (x axis) and normal distribution (y axis). The pink circles correspond to FSRQs, while blue triangles stand for BL Lacs. The green line corresponds to the condition $\mathrm{y}=\mathrm{x}$. Right: computed skewnesss from the log-normal distribution (x axis), and normal distribution (y axis).
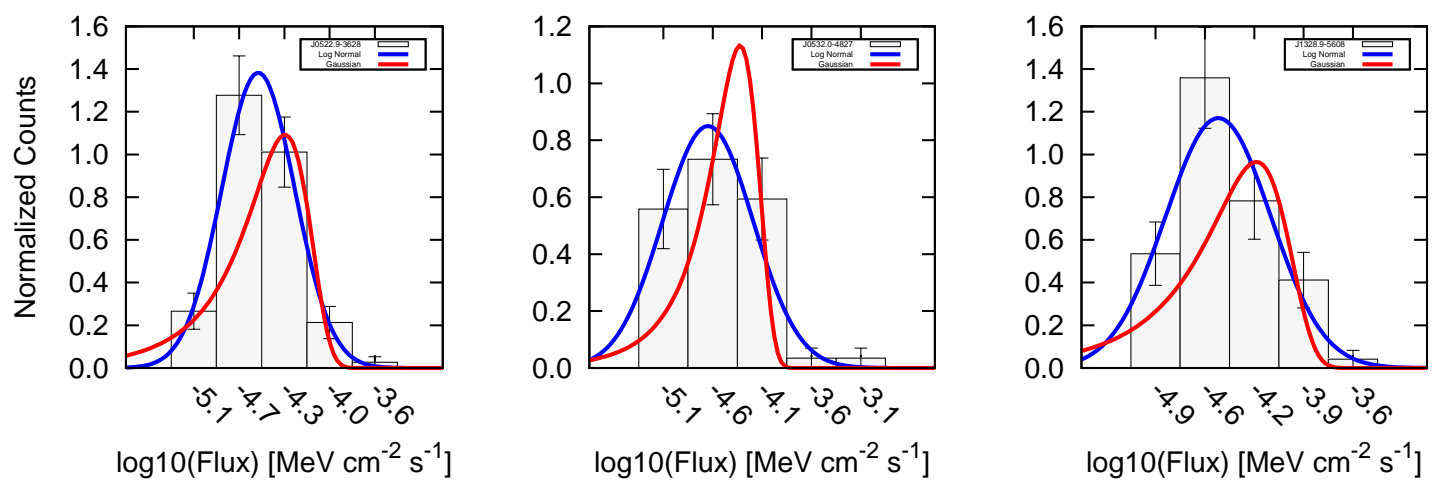

Fig. 3 Flux distribution of bright blazars in $\gamma$-ray band. The pointers are same as in the Fig. 1 


\section{ASSOCIATION OF FLUX DISTRIBUTION WITH SPECTRAL INDEX}

In order to investigate the association of flux variability with spectral indices, we estimated the monthly spectra of the blazars. All the considered blazars were well described by either simple power law or log parabola models. In order to compare the spectral indices of the spectra described by both models, it would be meaningful to calculate the spectral indices at a specific energy, which was chosen to be $1 \mathrm{GeV}$ (denoted by $\alpha_{1 \mathrm{GeV}}$ ). The spectral index at energy $\mathrm{E}$ is defined by

$$
\alpha_{E}=\alpha+2 \beta \log \left(E / E_{p}\right)
$$

where $\alpha$ is spectral index at pivot Energy $\mathrm{E}_{\mathrm{p}}$, and $\beta$ is the measure of spectral curvature. We have found that the average spectral indices for all FSRQs is $2.28 \pm 0.03$. However, the FSRQ J2254.0+1608 (a.k.a. 3C 454.3, which is the brightest blazar in $\gamma-$ ray band) shows a harder spectrum of index $1.72 \pm 0.06$. It has to be also noted that the spectral index of the steady FSRQ J0957.6+5523 is also smaller, which is $1.91 \pm 0.05$. The mean spectral indices of all other FSRQs fall in the range of 2.08-2.75. However, the mean spectral indices of BL Lacs fall in the range of 1.61-2.49, with a mean value of $2.01 \pm 0.11$.

The mean spectral indices of BCUs J0522.9-3628, J0532.0-4827 and J1328.9-5608 are 2.70, 2.64, and 2.74 respectively. These values fall in the range of spectral indices of other bright FSRQs, LBLs or IBLs, but comparatively higher than the spectral indices of HBLs.

The flux variability has been plotted against the spectral index in Fig 4. The HBLs (red square) fall in the left-low corner of the diagram, while FSRQs (pink circle) show a wider distribution. The IBLs (green triangle) and LBLs (gray dumbbell) fall in similar range of spectral index, though the variability distribution is wider in the case of former. The boxes represent the two-standard deviation uncertainty in $\alpha_{1 \mathrm{GeV}}$ and $\sigma$ from their respective mean value. The bright BCUs that were analyzed fall in the band of FSRQs. We also notice a slight correlation between the spectral index and standard deviation of the flux. The $\alpha_{1} \mathrm{GeV}$ and $\sigma$ of all blazars can be roughly fitted by a straight line of slope $0.24 \pm 0.04$, which is indicated by a blue dotted line in the figure 4 . The brightest BCUs show similar features as in the case of other blazars with respect to spectral index and flux distribution. All three BCUs (indicated as purple tilted square in fig. 4) fall beyond the 2-standard deviation uncertainty region (dotted box) of BL Lac sources. However, they are placed within the 2-standard deviation uncertainty region of FSRQ.

Additionally, we have also compared the monthly spectral indices of each source with their corresponding luminosity. It is interesting to note that every source shows harder when brighter phenomena. The monthly spectral indices and corresponding luminosity can be well fitted by a straight line. The average indices are found to be $-0.09+0.01$ for FSRQs, while it is $-0.11 \pm 0.01,-0.13 \pm 0.01,-0.13 \pm 0.01$ for HBL, IBL, and LBL respectively, suggesting the harder when brighter phenomena among the different bright blazar classes are not significantly different.

\section{DISCUSSION}

After a detailed study on the $\gamma$-ray flux of 38 brightest blazars, we found that the flux distributions predominantly suggest log-normal distribution rather than a normal distribution. We verified the log-normality (over normality) using both reduced $\chi^{2}$ and AD-test. The log-normality was rejected only in the case of three (in a 


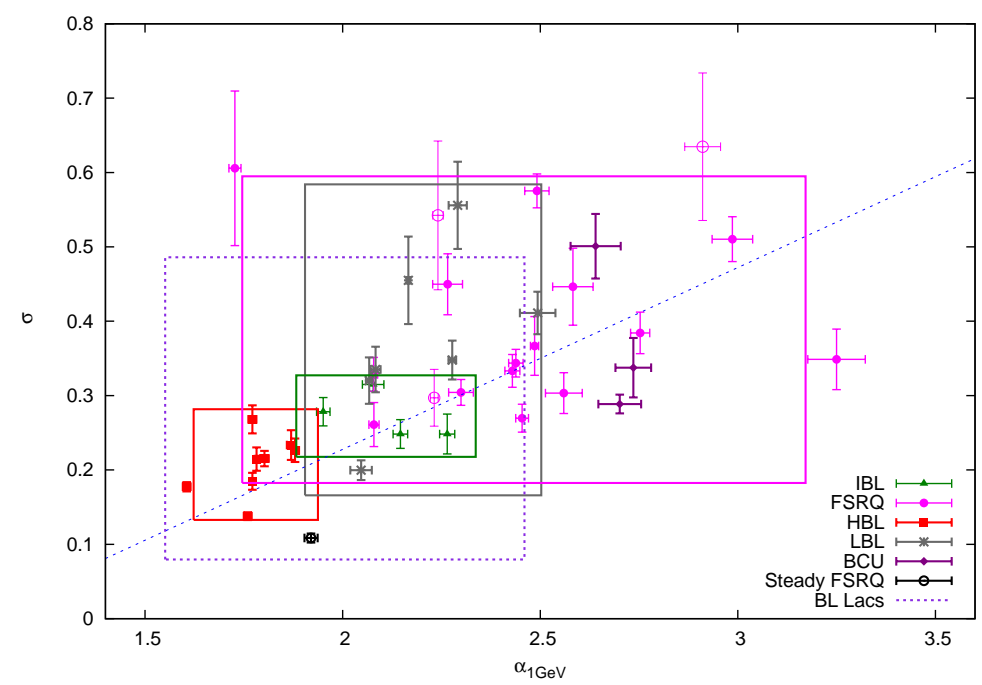

Fig. 4 The standard deviation $(\sigma)$ of the blazar flux distribution vs their corresponding average spectral index at $1 \mathrm{GeV}\left(\alpha_{1 \mathrm{GeV}}\right)$. Each blazar class has been marked with different pointers. HBL: red filled square, IBL: green triangle, LBL: gray dumbbell, FSRQ: pink circle, BCU: purple tilted square. The FSRQs which reject the log-normal flux distribution has been marked as pink open circle. The steady FSRQ has been shown separately black filled circle. The rectangle boxes correspond to two-standard-deviation uncertainty from the mean of $\sigma$ and $\alpha_{1 \mathrm{GeV}}$ for each blazar class. The color of the box is chosen to be same as that of pointers for each blazar class. The blue-violet dotted box corresponds to two-standard-deviation uncertainty from the mean of $\sigma$ and $\alpha_{1 \mathrm{GeV}}$ for all BL Lac sources

sample of 38) blazars. However, the normal distribution was rejected for all blazars (except J0957.6+5523, though the reduced $\chi^{2}$ was high). The flux distribution of the three brightest BCUs follow log-normal distribution. From the obtained spectral index and the flux standard deviation parameters, they fall beyond the 2-standard deviation uncertainty limits of HBL, IBL and LBL. Though it can not be asserted, we are tempted to associate these sources with FSRQs.

The log-normal distribution of the observed flux indicates the perturbation associated with the emission process to be of multiplicative nature rather than additive (Lyubarskii 1997; Arévalo \& Uttley 2006). Flux variation in blazars can be attributed to the complex interplay between the intrinsic and source parameters. A simple scenario is to associate the flux variation with the fluctuation in the emitting electron number density or the magnetic field. However, the linear dependence of the these quantities with the differential flux suggests, this will cause a normal flux distribution contrary to the observations. Alternatively, the particle acceleration and the diffusion processes can modify the shape of the emitting electron distribution (Kirk et al. 1998) and hence can be accounted for various flux distributions, including a log-normal one. The flux variation can also be associated with the change in the emission region geometry. Even though the change in volume associated with this can only produce normal flux distribution, inclusion of light travel time effects can significantly modify the same (Chiaberge \& Ghisellini 1999). However, the timescales associated with these processes are too short and hence will not reflect the log-normal distribution obtained in our study, where we used monthly averaged fluxes. 

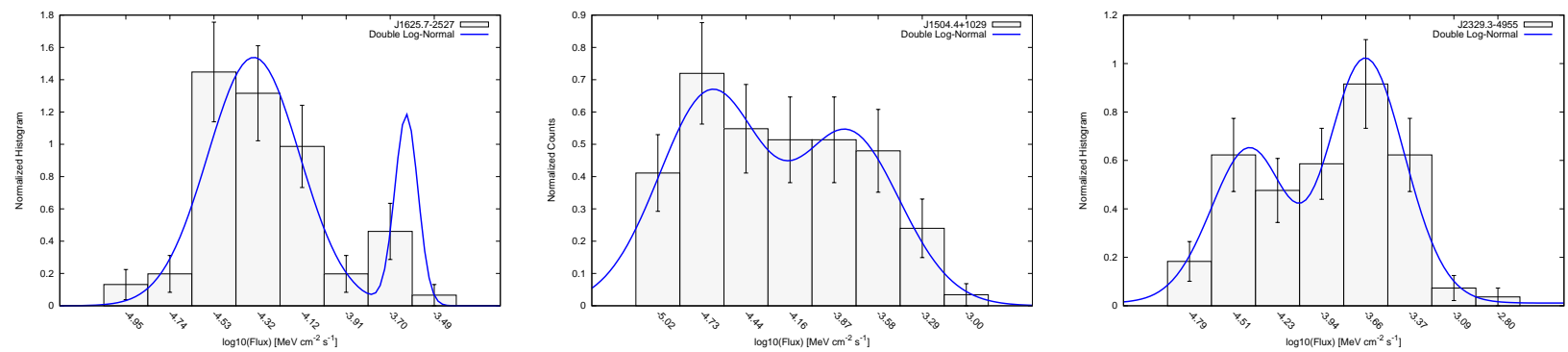

Fig. 5 Double log-normal fit to the flux distribution of the blazars J1625.7-2527, J1504.4+1029, and J2329.3-4955, which reject log-normal distribution. Since the statistics is not significant enough, these fits should be taken only as indicative.

A log-normal flux distribution can directly hint the linkage of blazar jet with the accretion phenomena since the latter is well proven to produce such distribution through the study of galactic X-ray binaries (XRBs)(Uttley \& McHardy 2001). The fluctuations in the disk at different radii are known to be produced independently by viscosity fluctuation on local viscous time scales, which modulates the mass accretion rate at larger distances from black-hole. The accretion rate variations then propagate to small radii through accretion flow and concoction of variations at different radii results in multiplicative emission. This model was put forward by Lyubarskii (1997) for explanation of observed X-ray variability time-scales in XRBs. Also, for non beamed accreting objects the variability timescales are found to be proportional to $M / \dot{\mathrm{m}}$, where $\mathrm{M}$ is mass of black hole and $\dot{m}$ is accretion rate (Körding et al. 2007). McHardy (2008) had found that same relation surprisingly holds even for beamed jet emission from blazars e.g, 3C 273, which should have otherwise shorter observed variability timescale due to relativistic time dilation than the timescale predicted using the black hole mass and accretion rate. Consequently, this lead to inference that source of variations in blazars lie out-side the jet i.e, in the accretion disks which then modulates the jet emission. A detailed study of month scale averaged flux distribution of blazars can hence be a key to understand disk-jet connection.

On the contrary to the interpretations above, a log-normal flux distribution can also arise from additive processes under specific conditions. For example, if the blazar jet is assumed to be a large collection of mini-jets, then the logarithm of composite flux will show a normal distribution (Biteau \& Giebels 2012).

We note that the AD statistics does not reject the normality of flux distribution of J0957.6+5523. Moreover, the standard deviation obtained from the flux distribution of this source exhibits a significant difference from that of other blazars. 3LAC labeled this source as an FSRQ, based on the presence of broad optical emission lines, large redshift and high $\gamma$-ray luminosity of the order of $\approx 10^{47} \mathrm{erg} \mathrm{s}^{-1}$. However, the integrated spectrum and morphological properties obtained from the VLBA observations question the FSRQ classification of the source, and suggest it as one of the weakest Compact Symmetric Object (Rossetti et al. 2005). Moreover, the brightness temperature of this source was found to be significantly lower $\left(2 \times 10^{8} \mathrm{~K}\right.$ at $5 \mathrm{GHz}$, Taylor et al. 2007), than that of other $\gamma$-ray blazars (McConville et al. 2011). These studies, together with our results, suggest that more multi-wavelength studies are required before associating this source to an FSRQ. 

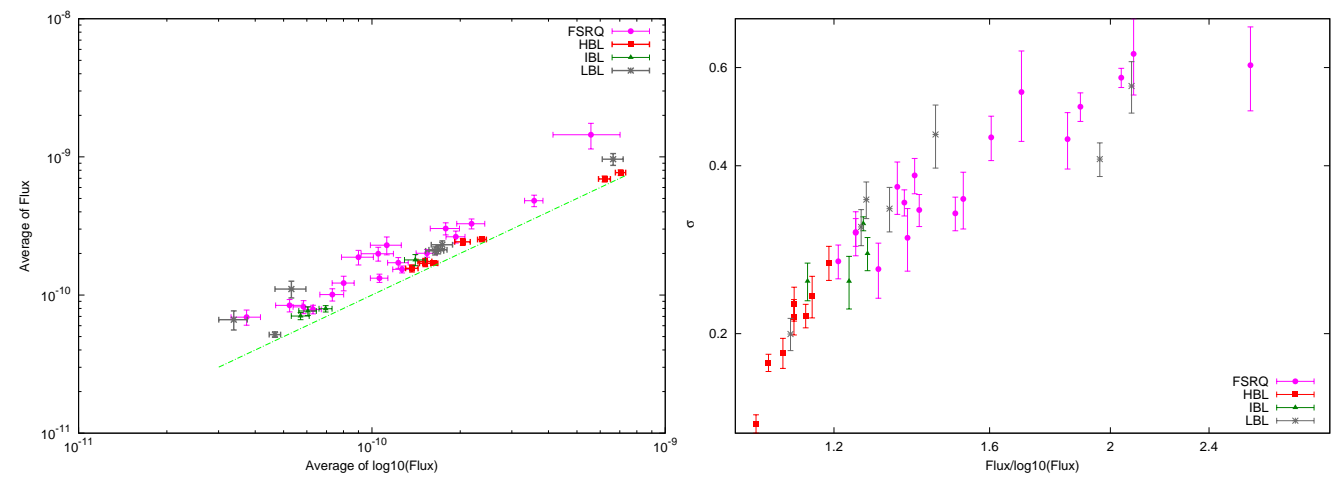

Fig. 6 Left plot shows the average of flux vs average of logarithm of flux of bright blazars. The line shows the condition of equality of quantities. Standard deviation of the log-normal flux distribution is plotted against the ratio between flux and logarithm of flux. Pointer color indicates the blazar class, pink: FSRQ, red: HBL, green: IBL, and gray: LBL.

We have also investigated the possibility of double log-normal distribution of fluxes for the sources that reject log-normal distribution both in $\chi^{2}$ and AD test (J1625.7-2527, J1504.4+1029, and J2329.3-4955). The two distinct log-normal profiles may indicate different flux states correspond to a low and high states of the source (Kushwaha et al. 2016). It is interesting to note that the flux distribution of all three sources exhibit hints of double log-normal distribution (Fig. 5). However, the statistics of this distribution is not significant enough, hence these result should be taken only as an indicative.

Another implication of our study is on the averaging of long term flux. We recommend the usage of the average of flux in log scale, rather than estimating average flux in a linear scale, especially for the highly variable sources. We show the difference of averaged flux in both linear and log-scale in Fig 6. For example, in the case of FSRQs, the average value of Flux/ $\log _{10}$ (Flux) falls around $\sim 1.7$, while the maximum value (in the case of $3 \mathrm{C} 454.3$ ) goes up-to 2.8. These values imply that averaging flux over a linear scale will significantly overestimate the same, which would in turn gives rise to inaccurate SED non-thermal emission model parameters.

\section{CONCLUSION}

We studied in detail the flux distribution properties of 38 brightest $\gamma$-ray blazars using Fermi-LAT data of more than 8 years. The flux distribution suggest log-normal distribution, for 35 blazars, indicating a multiplicative perturbation associated with the emission process. Similar features were obtained also in the case of BCUs. On the other hand, the flux distributions of three FSRQs - J2329.3-4955, J1504.4+1029, and J1625.7-2527 - reject both log-normal and normal distribution. This could be due to two or more independent flux states associated with the source, however, more statistics is required to study these effects in detail. It would be also interesting to perform an elaborate study with better statistics for more blazars in $\gamma$-rays, and compare the properties with that of their X-ray counterparts. 


\section{ACKNOWLEDGEMENT}

ZS, SS and NI are thankful to Indian Space Research Organization program (ISRO-RESPOND) for the financial support under grant no. ISRO/RES/2/396.

\section{References}

Ackermann, M., Ajello, M., Atwood, W. B., et al. 2015, ApJ, 810, 14

Arévalo, P., \& Uttley, P. 2006, MNRAS, 367, 801

Atwood, W. B., Abdo, A. A., Ackermann, M., et al. 2009, ApJ, 697, 1071

Band, D. L., \& Grindlay, J. E. 1985, ApJ, 298, 128

Biteau, J., \& Giebels, B. 2012, A\&A, 548, A123

Blandford, R. D., \& Königl, A. 1979, ApJ, 232, 34

Blandford, R. D., \& Znajek, R. L. 1977, MNRAS, 179, 433

Böttcher, M. 2007, Ap\&SS, 309, 95

Chiaberge, M., \& Ghisellini, G. 1999, MNRAS, 306, 551

Dermer, C. D., Schlickeiser, R., \& Mastichiadis, A. 1992, A\&A, 256, L27

Fan, J. H., Yang, J. H., Liu, Y., et al. 2016, ApJS, 226, 20

Gaskell, C. M. 2004, ApJ, 612, L21

Giebels, B., \& Degrange, B. 2009, A\&A, 503, 797

Kirk, J. G., Rieger, F. M., \& Mastichiadis, A. 1998, A\&A, 333, 452

Körding, E. G., Migliari, S., Fender, R., et al. 2007, MNRAS, 380, 301

Kushwaha, P., Chandra, S., Misra, R., et al. 2016, ApJ, 822, L13

Kushwaha, P., Sinha, A., Misra, R., Singh, K. P., \& de Gouveia Dal Pino, E. M. 2017, ApJ, 849, 138

Lyubarskii, Y. E. 1997, MNRAS, 292, 679

Lyutyj, V. M., \& Oknyanskij, V. L. 1987, AZh, 64, 465

Mannheim, K., \& Biermann, P. L. 1992, A\&A, 253, L21

Marscher, A. P., \& Gear, W. K. 1985, ApJ, 298, 114

McConville, W., Ostorero, L., Moderski, R., et al. 2011, ApJ, 738, 148

McHardy, I. 2008, in Blazar Variability across the Electromagnetic Spectrum, 14

Rossetti, A., Mantovani, F., Dallacasa, D., Fanti, C., \& Fanti, R. 2005, A\&A, 434, 449

Shah, Z., Sahayanathan, S., Mankuzhiyil, N., et al. 2017, MNRAS, 470, 3283

Sikora, M., Begelman, M. C., \& Rees, M. J. 1994, ApJ, 421, 153

Sinha, A., Shukla, A., Saha, L., et al. 2016, A\&A, 591, A83

Taylor, G. B., Healey, S. E., Helmboldt, J. F., et al. 2007, ApJ, 671, 1355

Tluczykont, M., Bernardini, E., Satalecka, K., et al. 2010, A\&A, 524, A48

Urry, C. M., \& Padovani, P. 1995, PASP, 107, 803

Uttley, P., \& McHardy, I. M. 2001, MNRAS, 323, L26

Uttley, P., McHardy, I. M., \& Vaughan, S. 2005, MNRAS, 359, 345

Vaughan, S., Edelson, R., Warwick, R. S., \& Uttley, P. 2003a, MNRAS, 345, 1271

Vaughan, S., Fabian, A. C., \& Nandra, K. 2003b, MNRAS, 339, 1237 